\title{
DIRECT MEASUREMENT OF CRYSTAL-FIELD SPLITTINGS IN PrN*
}

\author{
H. L. Davis and H. A. Mook \\ Sol id State Division, Oak Ridge National Laboratory \\ Oak Ridge, Tennessee, 37830
}

\section{ABSTRACT}

Inelastic neutron scattering has been employed to measure the crystal-field splittings of the ${ }^{3} \mathrm{H}_{4}$ ground multiplet corresponding to the $\mathrm{Pr}^{3+}$ ion in $\mathrm{PrN}$. An analysis of the resulting data places the $\Gamma_{4}$ crystal-field level at $27.0 \pm 1.0 \mathrm{meV}$, the $\Gamma_{3}$ at $46.3 \pm 1.0 \mathrm{meV}$, and the $\Gamma_{5}$ at $91 . \pm 2$. meV, above the ground $\Gamma_{1}$. These results can be quantitatively accounted for by a point-charge model with a charge of -3 , which sharply contrasts with previous work of others that estabi ished the crystal-field levels in other PrX compounds ( $X=P$, As, $\mathrm{Sb}, \mathrm{Bi}, \mathrm{S}, \mathrm{Se}, \mathrm{Te}$ ) could be accounted for by point charges of -2 . This. difference between $\operatorname{PrN}$ and the other PrX compourids. strongly implies some major difference in their electronic structures, which may indicate their being, respectively, semicunducting and metallic.

\section{INTRODUCTION}

The compound PrN belongs to the large class of $\mathrm{NaCl}$-structured rare-earth monopnictides ( $N, P, A s, S b, B i)$ and monochalcogenides ( $S, S e$, Te). Generally, this class contains a diversity of different magnetic behaviors due to the presence of the rare-earths' $4 f$ electrons, with some compounds being ferromagnetic, others ferrimagnetic, while others are antiferromagnetic. But as is the case for the $\mathrm{Pr}$ compounds, not all the compounds are found to undergo magnetic ordering, presumably. due to crystal-field effects separating off a nonmagnetic ground level. Be it as it may, irregardless of whether a given compound undergoes magnetic ordering or not, its magnetic properties are profoundly affected by crystal-field effects. Thus, some quantitative information regarding the crystal-field effects in these compounds is important for understanding their magnetic properties.

Since the vast majority of the compounds in the above class have a basically metallic behavior, the standard photon spectroscopy methods, which have been useful for crystal-field studies in insulators, are of little value for the rare-earth compounds. Thus, most previous attempts at extracting crystal-field information for these compounds has been concerned with model analyses of "broad-band-spectral" data, such as specific-heat and/or susceptibility data. A markedly promising exception to the broad-band methods has been the application of neutron spectroscopy. For example, such techniques have been applied to all the PrX compounds except $X=N$ by Turberfield et al. 1 (TPBB) and to TmSb by Birgeneau et ar. 2

Research sponsored by the U. S. Atomic Energy Commission under contract with Union Carbide Corporation. 
For the considered rare-earth compounds, the rare-earth sitesymmetry is $o_{h}$. Thus, following the notation of Lea et al. 3 (LLW), the crystal-field Hamiltonian may be written

$$
H=W\left[[x / F(4)] 0_{4}+[(1-|x|) / F(6)] 0_{6}\right\},
$$

with $W$ being an overall splitting factor and $x$ related to the ratio of fourth-and sixth-order terms. From LLW it is seen the ninefolddegenerate ${ }^{3} \mathrm{H}_{4}$, the ground $\mathrm{J}$-multiplet of $\mathrm{Pr}^{3+}$, will split into two triplets $\Gamma_{5}$ and $\Gamma_{4}$, one doublet $\Gamma_{3}$, and one singlet $\Gamma_{1}$. The energetic ordering of these $\Gamma_{i}$ depends on the $x$ value representative of the considered solid; however, a simple point-charge model indicates for PrN they would order, with increasing energy, in the sequence $\Gamma_{1}$ $\Gamma_{4}, \Gamma_{3}$, and $\Gamma_{5}$. Indeed, this is the ordering found by TPBB on the $\mathrm{Pr}$ compounds they investigated, and it is the order reasonably expected for PrN. At the same time, bulk magnetic data does indicate $\Gamma_{1}$ is the ground level for PrN, but a disagreement exists concerning the $W$ and $x$ values which best reproduce PrN's susceptibjlity and specific heat data. For example, the work of Junod et al. 4 gives $x=-0.8$ and $W=0.62 \mathrm{meV}$, while Stutius ${ }^{5}$ estimates $x=-1.0$ and $W=1.76 \mathrm{meV}$. Thus, application of neutron spectroscopy to PrN should provide information concerning which values of $x$ and $W$ are more realistic.

The cross-section for scattering ${ }_{6}$ neutrons from a single $\mathrm{J}$ multiplet follows the proportionality ${ }^{6}$

$$
\left(\partial^{2} \sigma / \partial \Omega \partial \omega\right) \sim\left(k_{f} / k_{i}\right) F^{2}(\vec{Q}) \sum_{n m} \rho_{n} \mid\left\langle r_{i}\left|\vec{J}_{\perp}\right| m>\left.\right|^{2} \delta\left(\varepsilon_{n m}-\hbar \omega\right\rangle,\right.
$$

which is valid for small momentum transfers. The states $|n\rangle$ and $|m\rangle$ are crystal-field levels having an energy difference $\varepsilon_{n m}$, with the delta-function indicating the scattering neutron will undergo a gain or loss, $\hbar w$, in its kine'cic energy depending on the sign of $\varepsilon_{n m}$ :
Also, $J \mathbb{J}$ is the component of $J \perp$ to the scattering vector $\downarrow$, $F$ is the form factor, and $k_{j}$ and $k_{f}$ are the momentums of the incident and scattered neutrons. It is important to note, to first order, the relative scattering intensities for the allowed tiw are proportional to $\rho_{n}|<n| \vec{J}_{1}|m>|^{2}$, with $\rho_{n}$ baing a Boltzmann population factor. This proport tonality provides a valuable tool for interpreting the neutron data, since any resulting interpretation must provide consistency with any observed changes of intensity with temperature and allowed tiw. In this aspect, the recent documentafion of the $|<n| \vec{J}_{1}|m>|^{2}$ for the LLW Hamiltonian provided by Birgeneau is a valuable tool in the preliminary analysis of neutron crystal-field data.

\section{RESULTS AND DISCUSSION}

Our experiments used the Oak Ridge magnetically-pulsed time-offlight spectrometer, 8 which is capable of producing pulsed monochromatic neutron beams over a wide energy range. For example, in our study of PrN we have utilized beams of energies 13.1,36.7,69.8, and $91.6 \mathrm{meV}$. Then, by using cross-correlation applied between the pulsing signal and the time distribution of neutrons arriving at a given 
detector, the desired data relating scattered neutron counts vs. time-of-flight channel (or energy) is readily obtained.

The PrN samples consisted of about $3 \mathrm{cc}$. of powder, and were kindly furnished by D. E. LaValle.9 Results obtained at hellum temperature when using $36.7 \mathrm{meV}$ incident neutrons are shown in Fig. 1 . This figure is a plot of scattered neutron counts vs, energy of the time-of-flight channel. The energies plotted in this and later

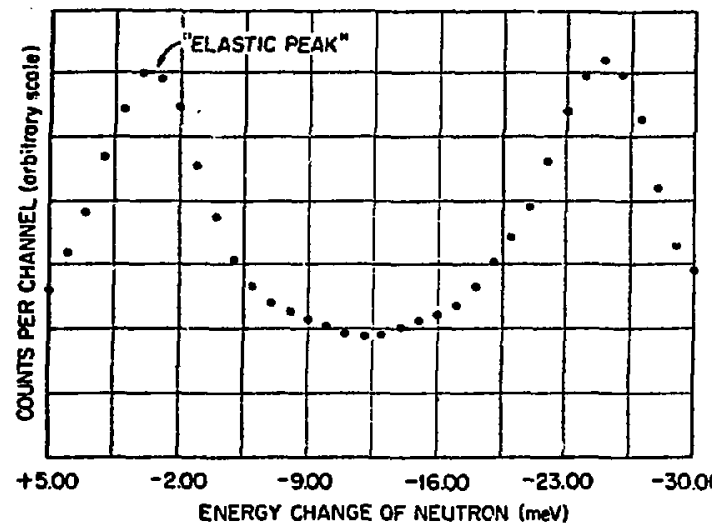

Fig. 1. Data for PrN at helium temperature.

ifigures correspond to the average times of the time channels, with a positive (negative) energy representing a neutron gaining (losing) that energy. Also; due to the $\left(k_{f} / k_{q}\right)$ factor of $E q$. (2) and the channels' energy widths being proportional to $\left(k_{f} / k_{j}\right)^{3}$, the results of Fig. 1 represent raw data after multiplication by a factor $\left(k_{i} / k_{f}\right)^{4}$ and being averaged over the resolution width of the spectrometer. Results obtained at room temperature, using an incident neutron energy of $13.1 \mathrm{meV}$, are displayed in Fig. 2; here raw data is p'lotted without any $\left(k_{j} / k_{f}\right)^{4}$ or resolution corrections.

The helium temperature results of Fig. I are easily and directly analyzed, since only excitation transitions from the ground $\Gamma_{1}$ will be possible. Also, because the only al lowed transition with $\Gamma_{1}$ involves $\Gamma_{4}$, the energy loss peak in the vicinity of 26 meV corresponds to this transition. However, the $\Gamma_{1} \rightarrow \Gamma_{4}$ transition energy

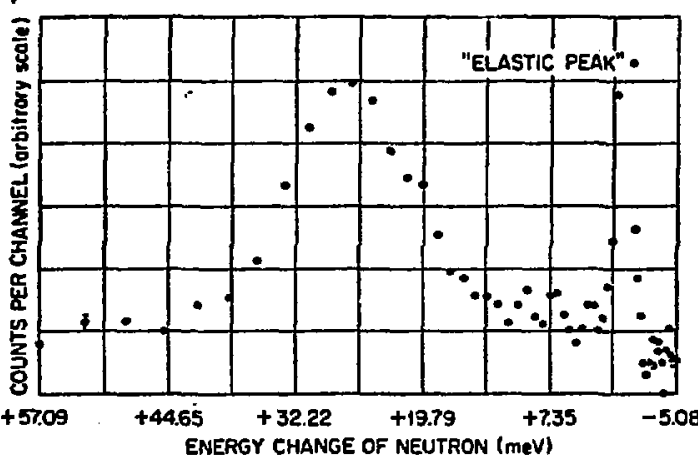

Fig. 2. Data for PrN at room temperature. will be slightly greater than the energy of the peak maximum due to the $F^{2}$ (ख) factor in Eq. (2). Of course, for higher temperatures, transitions imolving both neutron energy loss and gain are possible and will have. intensities as dictated by the products $\rho_{n}|<n| \vec{J}_{1}|m>|^{2}$ of $E q$. (2). Two such energy gain transitions are contained in. the data of Fig. 2, with its peak maximum corresponding to the $\Gamma_{4} \rightarrow \Gamma_{1}$ transition. At the same time, there appears to be a shoulder on - the right of the major peak in Fig. 2. Since the LLW results show the ratio $\left(\Gamma_{4} \rightarrow \Gamma_{1}\right) /\left(\Gamma_{3} \rightarrow \Gamma_{4}\right)$ is independent of $x$ and equal to $7 / 5$, it is entirely reasonable to assign this shoulder to de-excitation from the $\Gamma_{3}$ to $\Gamma_{4}$. 
In order to test the above peak and shoulder assignment, we. have written a computer program based on the theoretical model described by. Eqs. (1) and (2). The procedures used were similar to, but not identical to, the ones described by TPBB, and basically involve varying parameters to obtain a "best fit". The parameters used are $W$ and $x$ of Eq. (1), and a level width to introduce an essumed gaussian smearing of the $\Gamma_{i}$ levels.. For a given set of these parameters the program calculates the cross-section of Eq. (2) as a function of $h \omega$, and then convolutes the cross-section results with the - measured instrumental resolution which varies with hw. These results are then integrated over the energy-widths for each of the considered channels. The final result of. this process is relative values of theoretical counts per channel, and these can be compared with experimental counts after subtraction of a parametric background value. which is assumed constant for each considered channel. The results of using the program to obtain the best rms deviation to some of the data of Fig. 2 is shown in Fig. 3(a). The rms deviation between the calculated and experimental points of Eig. $3(a)$ is less than the statistical counting error which is indicated in Fig. 2. Also, the fit iliustrated by Fig. $3(a)$ leads to the energies quoted for the $\Gamma_{4}$ and $\Gamma_{3}$ leveis in Fig. $3(b)$, which should be considered to have enperimental errors of, roughly, $\pm 1.0 \mathrm{meV}$.

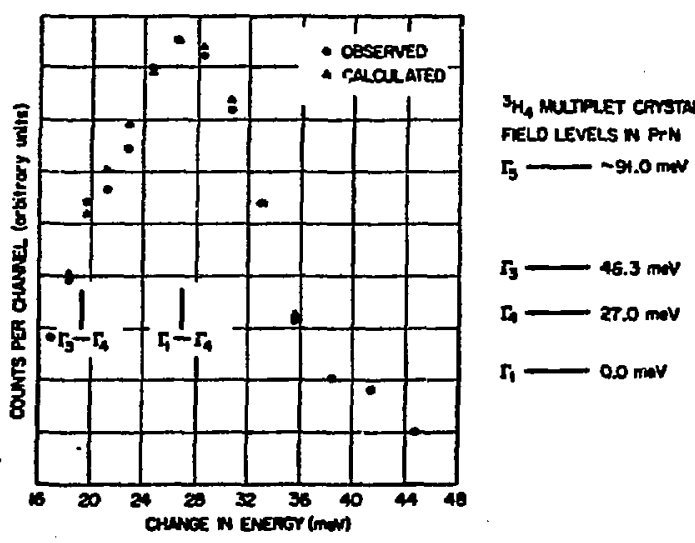

Fig. 3. (a). Comparison of PrN data at room temperature with calculated results. (b). The energy-level diagram for PrN following from our analysis. tempted detailed intensity calculations involving the $\Gamma_{5}$, qualitative intensity considerations are consistent with out assignment.

The energy values of Fig. 3(b) may be generated, within the quoted error 1 imits, by use of $L L W$ parameters $x=-0.97$ and $W=1.68$ meV. These direct neutron spectroscopy values are quite close to the values estimated by Stutius ${ }^{5}$ of $x=-1.0$ and $W=1.76$ meV from insufficient to place the remaining $\Gamma_{5}$ of the ${ }^{3} \mathrm{H}_{4}$ multiplet, which can have transitions with the $\Gamma_{3}$ and $\Gamma_{4}$. In order to place the $\Gamma_{5}$ we have used data which were obtained at room temperature using incident neutron results of 69.8 and $91.6 \mathrm{meV}$. The only. pert inent information these data contain, not al ready contained in Figs. I or 2 , are a weak peak corresponding to neutrons losing $\approx 45 \mathrm{meV}$ with use of the $69.8 \mathrm{meV}$ beam and a strong shoulder-type indication of energy loss at $\approx 65 \mathrm{meV}$ with : the 91.6 bean. These results are entirely consistent with placing the $\Gamma_{5}$ at $91 \pm 2 \mathrm{meV}$, which would give a $\Gamma_{3}+\Gamma_{5}$ transition at $\approx 45$ $\mathrm{meV}$ and $a \Gamma_{4} \rightarrow \Gamma_{5}$ at $\simeq 65 \mathrm{meV}$. Also, although we have not at-

The data of Figs. 1 and 2 is 
specific heat data; thus we conclude the values $x=-0.8$ and $W=$ 0.62 estimated by Junod et $a Z .^{4}$ do not realistically reflect the actual crystal-field of PrN. At the same time, our value of $27.0 \pm$ $1.0 \mathrm{meV}$ for the $\Gamma_{4}$ is entirely consistent with the value $27.2 \mathrm{meV}$ tabularly quoted by Bucher et az. 10 as having been obtained by neutron spectroscopy. Although no details of their data have been reported, it is our impression Bucher et al. were only able to detect the $\Gamma_{4}$ level.

The $W$ and $x$ values we have obtained for PrN are surprisingly close to the values $x=-0.96$ and $W=1.69 \mathrm{meV}$, which would be predicted by a nearest-neighbor point-charge model with charges of -3 . To obtain this result we have used the nonrelativistic Hartree-Fock radial integrals $\left\langle r^{n}\right\rangle$ tabulated by Freeman and Watson 11 for $\mathrm{Pr}^{3+}$. Al though relativistic effects can be very significant in the $\left\langle r^{n}\right\rangle$ integrals, we have used the Freeman and Watson values in order to easily compare our result with the point-charge analys is of TPBB on the other $\operatorname{Pr}$ compounds since they used the same $\left\langle r^{n}\right\rangle$ values. That is, the main point we want to make here is not that our PrN results have an apparent agreement with a point-charge model, which they would not if relativistic $\left\langle r^{n}\right\rangle$ were used, but that the Prin results do not foliow the same trend as found for the other $\operatorname{PrX}$ compounds. This is immediately seen from the fact that TPBB found that the same pointcharge model we have used to obtain a charge of -3 for PrN requires a charge of -2 for $\operatorname{PrX}(x=P, A s, S b, B i, S, S e, T e)$. Thus, we conclude that this difference in model charge value implies some major difference between the overall electronic structure of PrN and that of the other PrX compounds. We suggest this difference is that PrN is indeed an intrinsic semiconductor as has been implied by the work, e.g., of Sclar, 12 while the other PrX compounds are intrinsically metallic.

\section{REFERENCES}

1. K. C. Turberfield, L. Passel1, R. J. Birgeneau and E. Bucher, J. App1. Phys. 42, 1746 (1971).

B. R. J. Birgeneau, E. Bucher, L. Rassell and K. C. Turberfield, Phys. Rev. B 4, $718 \cdot(1971)$.

3. K. R. Lea, M. J. M. Leask and W. P. Wolf, J. Phys. Chem. Sol ids 23, 1381 (1962).

4. $\overline{\mathrm{P}}$. Junod, A. Menth and 0. Vogt, Phys. Kondens. Materie 8, 323 (1969).

5. W. Stutius, Phys. Kondens Materie 10, 152 (1969).

6. P. deGennes, Magnet ism, G. T. Rado and H. Suhl, eds. (Academic Press, New York, 1963), Vol. 3, p. 115.

7. R. J. Birgeneau, J. Phys. Chem. Sol ids 33, 59 (1972).

8. H. A. Mook and M. K. Wilkinson, p. 173 in Proceedings on Instrumentation for Neutron Inelastic Scattering Research, Vol. III, IAEA, VIenna, 1970.

9. D. E. LaValle, Analytical Chemistry Division, ORNL.

10. E. Bucher, K. Andres, J. P. Maita, A. S. Cooper and L. D. Longinotti, J. Phys. (Paris) 32, 114 (1971)

11. A. J. Freeman and R. E. Watson, Phys. Rev. 127, 2058 (1962).

12. N. Sclar, J. Appl. Phys. 33, 2999 (1962); 35, 1534 (1964). 